limits and aorta were less in benign RPF, however, significant differences were only observed when comparing with lymphoma ( $p$ values: all $<0.001$ ) but not with metastatic carcinoma ( $p$ value: $0.396,0.181,0.112$ and 0.64 ). A greater frequency of retroperitoneal lesions with high FDG-uptake (100\% vs $77.5 \%$, p value: 0.017$)$ and a higher mean SUVmax (12.2 vs $4.8, p$ value: $<0.001)$ were observed in malignant RPF. The frequencies of LNs with high FDG-uptake were greater with significance in malignant RPF except for hilar/mediastinal and cervical LNs, hence the rest LNs were regarded as specific LNs. At ROC analyses, the AUCs of SUVmax and specific LNs were 0.893 and 0.947 . The sensitivity and specificity were $85.7 \%$ and $81.4 \%$ when the SUVmax was 6.23 . The AUC of logistic regression model combining SUVmax and specific LNs was 0.974 with sensitivity of $90.5 \%$ and specificity of $90.1 \%$.

Conclusions: PET/CT could help distinguish benign from malignant RPF, especially when taking into account the FDG-uptake of retroperitoneal process and LNs.

Disclosure of Interest: None declared

DOI: 10.1136/annrheumdis-2017-eular.2588

\section{FRI0305 RELATIVE FDG ACCUMULATION OF THE AORTIC WALL LESIONS TO AORTIC BLOOD POOL IN 18F-FDG-PET AND PET/CT COULD BE A USEFUL PARAMETER FOR THE PREDICTION OF DISEASE RELAPSE AFTER SUCCESSFUL TREATMEN IN TAKAYASU ARTERITIS}

A. Ihata ${ }^{1}$, Y. Kunishita ${ }^{2}$, K. Minegishi ${ }^{2}$, R. Yoshimi ${ }^{2}$, Y. Kirino ${ }^{2}$, H. Nakajima ${ }^{2}$ ${ }^{1}$ Department of Rheumatology, Yokohama Medical Center/ National Hospital Organization; ${ }^{2}$ Department of Stem Cell and Immune Regulation, Yokohama City University Graduate School of Medicine, Yokohama, Japan

Background: The assessment of disease activity of Takayasu arteritis (TA) is difficult if symptoms and serum inflammatory marker were not detected. Even in those conditions, relapses were frequently observed during the dose reduction of corticosteroid and immunosuppressant. There is accumulating evidence that 18F-fluolodeoxyglucose-positron emission tomography (FDG-PET) and PET/ computed tomography (PET/CT) is useful for monitoring patients with TA when TA was clinically active. However, it is not clear the significance of FDG accumulations when TA was inactive.

Objectives: To investigate a quantitative predictor in FDG-PET or PET/CT scans for the relapse of TA.

Methods: We retrospectively investigated 76 FDG-PET or PET/CT scans and extracted 37 scans which were performed in inactive status. These scans were divided in two groups according to relapse of TA for 5years. The relapse was defined the increase of CRP and steroid dose or addition of immunosuppressant. FDG accumulations in aortic wall lesions of TA was evaluated by semi-quantitative index; the standardized uptake value (SUV). In addition to SUVmax in the aortic wall, we also calculated SUV ratio of maximum aortic wall uptake to mean lung uptake (ratio Lu), SUV ratio of maximum aortic wall uptake to mean liver uptake (ratio Li), and SUV ratio of maximum aortic wall uptake to mean aortic blood pool uptake (ratio BP). We compared groups using these parameters. We also determined the cutoff levels, sensitivity, and specificity of 4 sets of SUVs (SUVmax, ratio $\mathrm{Lu}$, ratio $\mathrm{Li}$, and ratio $\mathrm{BP}$ ) for the prediction of relapse using Receiver Operating Characteristic (ROC) analysis. Moreover, Kaplan-Mayer analysis for the long-term relapse-free survival was performed to assess the reliability of these cutoff levels.

Results:

Table 1. Characteristics of two groups

\begin{tabular}{lcc}
\hline Relapse & $(-)$ & $(+)$ \\
$\#$ & 17 & 20 \\
Age $(\mathrm{yr})$ & $47[30-72]$ & $28.5[14-68]$ \\
CRP $(\mathrm{mg} / \mathrm{dl})$ & $0.13 \pm 0.03$ & $0.13 \pm 0.03$ \\
Steroid dose (mg/d equivalent to prednisolone) & $15.6 \pm 2.4$ & $17.7 \pm 3.6$ \\
Immunosuppressant & $2 / 17$ & $7 / 35$ \\
Duration until relapse (Days) & & $702.5[4-1769]$ \\
\hline
\end{tabular}

In 37 total PET and PET/CT scan examinations, non-relapse and relapse groups included 17 and 20 scans, respectively. Relapse group had more immunosuppressant users than non-relapse group. Although CRP level and SUVmax were equivalent, ratio of SUV, especially ratio BP of relapse group was higher than that of non-relapse group $(p=0.09)$ (Figure top panel). The cut-off level of these parameters was calculated as follows; SUVmax 1.4, ratio Lu 5.31, ratio Li 1.01 , and ratio BP 1.41, respectively. Using these cut-off level, relapse rates of below and over cut-off level were as follows; SUVmax $50 \%$ vs $54 \%$, ratio Lu $43 \%$ vs $62 \%$, ratio Li $43 \%$ vs $69 \%$, and ratio BP $31 \%$ vs $67 \%$, respectively (Figure middle panel). Using Kaplan-Mayer analysis, relapse rate of these two groups divided by ratio BP was not significantly different $(p=0.268)$ though these two curves looked different (Figure bottom panel).

Conclusions: Our data suggest that ratio BP at stable condition, which represents relative FDG accumulation of the aortic wall lesions to aortic blood pool, could be a promising predictor to assess the relapse after successful treatment.

Disclosure of Interest: None declared

DOI: 10.1136/annrheumdis-2017-eular.6266
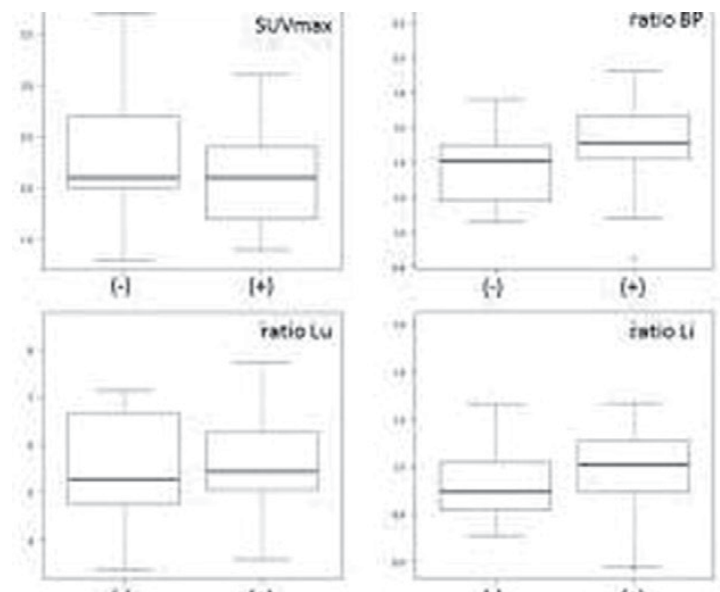

$(\cdot)$

[+]

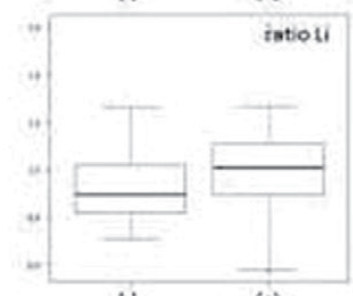

$\langle\rightarrow$

(

Correlotion between 4 parameters of FDG-PET and Relogse in Toksyssu srterits
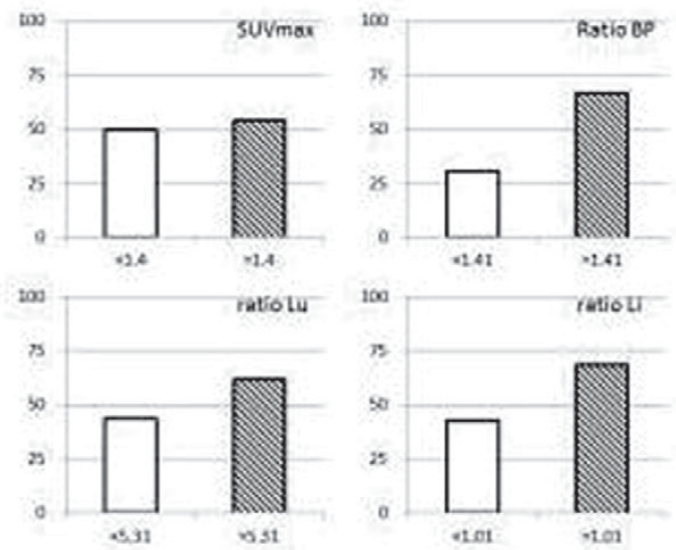

Rolopse rate between two voups divided by thresholds of 4 paramesers of $\&$ OG.PET

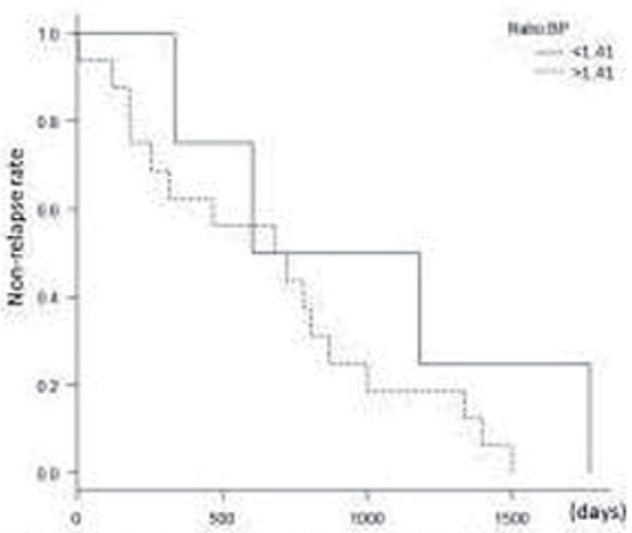

Kaplan-Neier analyas of relepse tate divided by Ratio 8 p theshold Abstract FRI0305 - Figure 1

\section{FRI0306 LONG TERM DRUG-FREE REMISSION IS FEASIBLE IN SEVERE BEHCET'S DISEASE AFTER CESSATION OF SUCCESSFUL ANTI-TNF TREATMENT: A SINGLE CENTER, RETROSPECTIVE LONGITUDINAL OUTCOME STUDY}

P.P. Sfikakis, A. Arida, S. Panopoulos, K. Fragiadaki, G. Pentazos, A. Laskari, M. Tektonidou, N. Markomichelakis. First Department of Propaedeutic and Internal Medicine and Joined Rheumatology Program, National and Kapodistrian University of Athens Medical School, Athens, Greece

Background: The efficacy of anti-TNF treatment for patients with severe forms of Behcet's disease (BD) is well established (ref), but long term data on the outcome after cessation of such treatment are lacking.

Objectives: To examine whether sustained long term remission of severe BD is feasible after cessation of successful anti-TNF treatment.

Methods: This retrospective longitudinal outcome study was conducted in December 2016 and included all patients with severe BD refractory to conventional immunosuppressive therapy who were considered complete responders to continuous anti-TNF treatment in our center, the first being treated in 2000 . 
Study's endpoint was the proportion of patients achieving sustained complete remission of $\mathrm{BD}$ for at least 3 years after cessation of the anti-TNF agent.

Results: A total of 28 patients in whom infliximab and/or adalimumab treatment was given, always combined with azathioprine unless not tolerated $(n=2)$, and discontinued anytime before December 2013 were eligible for analysis. Following cessation of successful anti-TNF treatment (median duration of 2 years) $13 / 28$ patients achieved the study's end-point. The main reason for anti-TNF administration was sight-threatening ocular disease $(n=12)$ or intestinal disease $(n=1)$. The remaining 15 patients relapsed within 1.5 year (main reason for anti-TNF: ocular disease, $n=9$; neuro-BD, $n=2$; severe mucocutaneous disease, $n=3$; intestinal disease, $n=1$; median treatment duration of 24 months); $12 / 15$ were successfully re-treated with anti-TNF. So far, 3 of them (ocular disease, $n=2$; neuro-BD, $n=1$ ) have achieved the study's end-point (median re-treatment duration of 2 years). Overall, our 16 patients who achieved the study's end-point (57\%) are in complete disease remission ranging from 3 to 12 years (5.7 years, median). Nine patients with severe ocular disease are currently any drug-free (32\%), whereas the 7 remaining patients are on low doses of conventional immunosuppressive therapy $(25 \%)$. Notably, those patients on drug-free remission had shorter median disease duration at initiation of anti-TNF treatment, compared to the remaining patients (1 versus 3 years, respectively).

Conclusions: Sustained drug-free remission for many years after cessation of successful anti-TNF treatment is feasible in some patients with severe BD. Since anti-TNF-induced "cure" can never be differentiated from a spontaneous remission by natural history, further studies should examine whether early anti-TNF treatment must be intended for every patient with severe BD. References:

[1] Hatemi G, et al. EULAR recommendations for the management of Behçet disease. Ann Rheum Dis. 2008 Dec;67(12):1656-62.

Disclosure of Interest: None declared

DOI: 10.1136/annrheumdis-2017-eular.5338

\section{FRI0307 AN OUTCOME SURVEY OF 100 PATIENTS WITH CEREBRAL VENOUS SINUS THROMBOSIS DUE TO BEHÇET'S SYNDROME FOLLOWED UP AT A SINGLE, DEDICATED CENTER}

E.A. Kurt ${ }^{1}$, Y. Ozguler ${ }^{1}$, N. Kocer ${ }^{2}$, D. Ucar ${ }^{3}$, U. Uygunoglu ${ }^{4}$, C. Islak ${ }^{2}$, S. Ugurlu ${ }^{1}$, G. Hatemi ${ }^{1}$, S. Saip ${ }^{4}$, M. Melikoglu ${ }^{1}$, I. Fresko ${ }^{1}$, V. Hamuryudan ${ }^{1}$, Y. Ozyazgan ${ }^{3}$, S. Yurdakul ${ }^{1}$, A. Siva ${ }^{4}$, H. Yazici ${ }^{1}$, E. Seyahi ${ }^{1}{ }^{1}{ }^{1}$ Department of Internal Medicine Division of Rheumatology; ${ }^{2}$ Department of Radiology; ${ }^{3}$ Department of Ophthalmology; ${ }^{4}$ Department of Neurology, Istanbul University Cerrahpasa Medical Faculty, Istanbul, Turkey

Background: Behcet's syndrome (BS) is a well-recognized cause of cerebral venous sinus thrombosis (CVST).

Objectives: We aimed to assess the outcome of a large cohort of patients with CVST due to BS attending a single dedicated center.

Methods: We identified 100 ( $81 \mathrm{M} / 19$ F) patients with BS who were diagnosed as having CVST. All contacted were called back to the outpatient clinic for clinical and imaging re-evaluation.

Results: The mean age of the patients at the onset of the symptoms was $28 \pm 10$ years. A total of 48 patients developed CVST before or at the onset of ISG fulfillment, while 52 developed CVST after a median 3 [2-8] years of ISG fulfillment. Superior sagittal $(n=47)$ and transverse sinuses $(n=46)$ were most commonly involved followed by sigmoid sinus $(n=26)$ and jugular vein thrombosis $(n=15)$. A total of $59(53 \mathrm{M} / 6 \mathrm{~F})$ patients had vascular involvement in addition to CVST: these were deep vein thrombosis of the lower extremities $(n=47)$, pulmonary artery involvement $(n=17)$, Budd-Chiari syndrome $(n=9)$, vena cava superior thrombosis $(n=6)$ and major arterial disease $(n=3)$. In about half $(32 / 59)$, CVST preceded any type of additional vascular involvement. Eye involvement was seen in 37, parenchymal CNS involvement in 8 (all later than CVST) and gastrointestinal involvement in 5 patients.

Seven patients had died. By the end of the study, 87 patients were alive and contacted with a median follow-up time of 11 [6-15] years. Only 6 patients had a relapsing CVST course.

Information about medical treatment was present in detail in 87 patients of whom 75 received short courses of glucocorticoids with $(n=12)$ or without anti-coagulants. A total of 81 patients received immunosuppressive agents, most commonly azathioprine. Four patients underwent lumbo-peritoneal shunting surgery (1 was successful) and 1 with arterio-venous fistula underwent vascular embolization.

Fifty patients were re-evaluated at the clinic. None had of symptoms of intracranial hypertension. Ophthalmological examination showed that 17 patients had complications such as bilateral optic atrophy $(n=3)$, bilateral papilledema $(n=5)$, bilateral optic disc pallor $(n=4)$ and fibrotic scars around optic disc $(n=5)$. Sensorineural type hearing loss was detected in 4 patients. Neurological examination was found to be normal in 43 patients with isolated CSVT, whereas abnormal in the remaining 7 patients with concomitant parenchymal CNS involvement.

Cranial MR/MR venographies at the end of follow-up, were abnormal in 36 patients showing occlusion/ irregularity/ hypoplasia or collaterals in the sagittal $(n=19)$ or transverse sinus $(n=17)$. In the remaining 14 , MR venographies were normal.

Conclusions: CVST due to BS is closely associated with vascular involvement in the body and may be considered as a risk factor for future vascular involvement. CVST relapses are rare; however, the course is not uneventful: visual acuity or field may be impaired totally or partially because of optic disc atrophy; in addition hearing deficits may occur.

Disclosure of Interest: None declared

DOI: 10.1136/annrheumdis-2017-eular.6323

\section{FRI0308 PREDICTORS OF HYPOGAMMAGLOBULINAEMIA IN RITUXIMAB TREATED PATIENTS. A RETROSPECTIVE ANALYSIS ON A MONOCENTRIC COHORT}

R. Padoan, M. Felicetti, M. Gatto, P. Polito, F. Ometto, D. Astorri, L. Friso, F. Cozzi, A. Doria, L. Punzi, F. Schiavon. Operative Unit of Rheumatology, Department of Medicine DIMED, University of Padova, Padova, Italy

Background: Rituximab (RTX), a chimeric monoclonal antibody against CD20, is increasingly used in the treatment of B-cell lymphomas and autoimmune conditions. It has been shown that some patients develop hypogammaglobulinaemia after treatment.

Objectives: To assess frequency and predictors of hypogammaglobulinaemia after RTX treatment in a monocentric cohort of patients affected with granulomatosis with polyangiitis (GPA), microscopic polyangiits (MPA) and connective tissue diseases (CTD).

Methods: We retrospectively reviewed all patients receiving RTX and concomitant/sequential immunosuppressants between 2007 and 2016 in a single rheumatologic center. Serum levels of total Ig and lymphocyte subsets were recorded at the time of RTX administration and 3-6 months later. We investigated the frequency of hypogammaglobulinaemia $(\mathrm{lgG}<6 \mathrm{~g} / \mathrm{L})$ and its related events. Results: 72 patients, 30 (41.6\%) GPA/MPA, $25(34.7 \%)$ systemic lupus erythematosus, $13(18.1 \%)$ systemic sclerosis and $4(5.6 \%)$ poly-dermatomyositis were treated with RTX. We analyzed 113 RTX infusions, with 41 (36.2\%) retreatments (median 2 [2-6]). We excluded 12 patients/18 infusions due to incomplete data. RTX was administered at the dosage of $1000 \mathrm{mg}$ twice in $68.1 \%$ of patients and $375 \mathrm{mg} / \mathrm{m}^{2}$ weekly in $31.9 \%$. IgG levels after RTX infusion were available in $68(71.6 \%)$ patients. We observed a significant decrease of IgG levels between baseline and 3-6 months after RTX infusion in all patients $(0.001)$. The frequency of patients with $\lg G<6 \mathrm{~g} / \mathrm{L}$ was $22.1 \%$, and $8.8 \%$ had $\lg G<4 \mathrm{~g} / \mathrm{L}$, significantly higher in GPA/MPA patients (0.008), with short disease duration (0.001), lower IgG levels at baseline (0.008), higher prednisone equivalent (PDE) cumulative dosage per year (0.006) and higher daily PDE dosage after RTX (0.001) (Table 1). After RTX, all patients had complete B-cells depletion.

At univariate analysis, $\lg \mathrm{g}<6 \mathrm{~g} / \mathrm{L}$ was associated with GPA/MPA diagnosis $(0.006$, OR 6 [1.5-24.2]), disease duration (<0.001, OR $0.2[0.1-0.9]$ ), RTX $375 \mathrm{mg} / \mathrm{m}^{2}$ weekly protocol ( $\mathrm{p}=0.017$, OR 4.1 [1.2-13.9]), PDE cumulative dosage per year $(<0.001$, OR 6.6 [1.3-33.6]), daily PDE intake $>15 \mathrm{mg} /$ day after $\operatorname{RTX}(<0.001$, OR 12.7 [3.1-52.5]) and lgG levels before RTX $(<0.001$, OR 18 [1.8-178]).

At multivariate analysis, daily PDE intake after RTX ( $>15 \mathrm{mg} /$ day) and GPA/MPA diagnosis resulted independent predictive factors for hypogammaglobulinaemia $(p=0.03$, OR 9.5, [2.2-41.7] and $p=0.43$, OR 4.7, [1.1-21.5]).

Patients affected with GPA/MPA were compared to CTD, as reported in Table 2. GPA/MPA patients at infusion were older (0.002), presented shorter disease duration (0.001), lower IgG levels at baseline $(<0.001)$ despite lower rate of nephrotic syndrome $(0.003)$. Moreover they were treated more frequently with Azathioprine $(0.007)$, RTX $375 \mathrm{mg} / \mathrm{m}^{2}$ weekly protocol $(<0.001)$ and higher PDE cumulative and daily dosage $(<0.001,0.01$ respectively).

Only 5 patients $(5.2 \%)$ experienced severe infections within 12 months, more frequently in $\lg G<6 \mathrm{~g} / \mathrm{L}$ patients $(0.007)$.

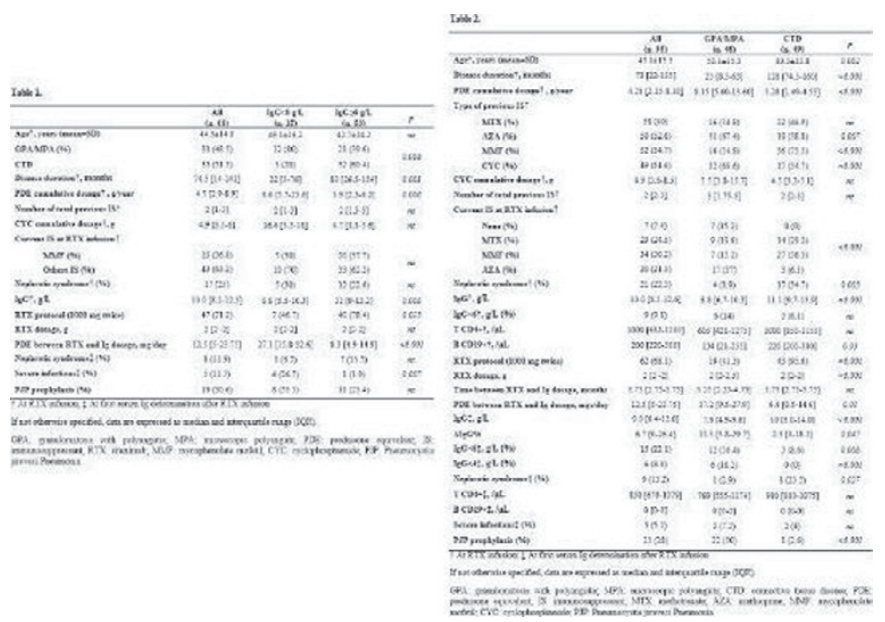

Conclusions: In autoimmune rheumatic diseases, diagnosis of GPA/MPA and glucocorticoid therapy resulted independent predictors of hypogammagobulinaemia after RTX treatment. Despite low IgG levels were associated with higher infections risk, rare severe infectious events were observed. 